\title{
Directional Ostwald ripening for producing aligned arrays of nanowires
}

Hye Jin Lee,,$^{\dagger}$ U-Jeong Yang, ${ }^{\dagger}$ Kyeong Nam Kim, ${ }^{+}$Soojin Park, $, ~ K y e ~ H y o u n g ~ K i l,{ }^{\perp}$ Jun Soo Kim, ${ }^{\perp}$ Alec M. Wodtke, ${ }^{\nabla}$ Won Jun Choi, ${ }^{O, *}$ Myung Hwa Kim, ${ }^{\perp, *}$ and Jeong Min Baik ${ }^{\dagger} * *$

†School of Materials Science and Engineering, Ulsan National Institute of Science and Technology

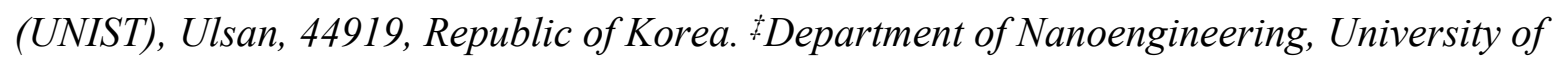
California, San Diego (UCSD), La Jolla, California 92093, USA. §Department of Chemistry, Pohang University of Science and Technology (POSTECH), Pohang, 37673, Republic of Korea. Department of Chemistry \& Nano Science, Ewha Womans University, Seoul, 03760, Republic of Korea. $\quad \nabla$ Department of Dynamics at Surfaces, Max Planck Institute for Biophysical Chemistry (MPI-BPC), Göttingen, 37077, Germany. ${ }^{\circ}$ Center for Opto-Electronic Materials and Devices, Korea Institute of Science and Technology (KIST), Seoul, 02792, Republic of Korea.

${ }^{*}$ Corresponding author E-mail: myungkim@ewha.ac.kr (MHK), wjchoi@kist.re.kr (WJC), jbaik@unist.ac.kr(JMB) 


\section{Experimental Section}

$\mathrm{VO}_{2}$ nanowire growth: For the growth of the $\mathrm{VO}_{2}$ nanowires, thin $\mathrm{V}_{2} \mathrm{O}_{5}$ films were initially deposited onto a $200 \mathrm{~nm}$ thick, thermally grown $\mathrm{SiO}_{2}$ layer on a $\mathrm{Si}(100)$ single crystal substrate in an $\mathrm{RF}$ reactive sputtering chamber with a Vanadium target (99.99\%), flowing argon/oxygen mixtures at an operating pressure of $2 \times 10^{-6}$ torr and substrate temperature of $500^{\circ} \mathrm{C}$. The thickness of the thin films was controlled in the approximate range $20 \mathrm{~nm}$ to $60 \mathrm{~nm}$ by adjusting the deposition time. The thin film was then transferred to the center of a horizontal quartz tube furnace to grow $\mathrm{VO}_{2}$ nanowire growth at atmospheric pressure. Nanowire growth was carried out for 3 hours at $650^{\circ} \mathrm{C}$ with flowing high purity $\mathrm{He}(99.999 \%)$ at $200 \mathrm{sccm}$.

Fabrication of $\mathrm{VO}_{2}$ nanowire array: $\mathrm{V}$-grooved $\mathrm{SiO}_{2} / \mathrm{Si}$ substrates were fabricated by potassium hydroxide $(\mathrm{KOH})$ etching. The native oxide on the Si (100) substrates was removed by buffered oxide etchant (BOE) and $500 \mathrm{~nm}$-thick $\mathrm{SiO}_{2}$ layer was deposited by using plasma-enhanced chemical vapor deposition (PECVD) to be used as a shadow mask for the wet etching process. By using a photomask having line patterns of pitch size from 2 to $6 \mu \mathrm{m}$ and conventional lithography process, line patterns of photoresist (PR) were obtained. By reactive ion etching (RIE) process, the $\mathrm{SiO}_{2}$ was selectively etched away, resulted in the formation of the line patterned $\mathrm{SiO}_{2}$ on $\mathrm{Si}$ substrate. The sample was dipped in $45 \%$ $\mathrm{KOH}$ solution for $1 \mathrm{~h}$ at $80^{\circ} \mathrm{C}$ to carry out the anisotropic wet etching, producing $\mathrm{V}$-groove templates with different angles of the grooves, as shown in Figure S5. By changing the etching time, the morphology of the templates could be also controlled. The etched sample was subsequently dipped in the diluted hydrofluoric acid $\left(\mathrm{HF}: \mathrm{H}_{2} \mathrm{O}=1: 1\right)$ at room temperature to remove the $\mathrm{SiO}_{2}$ etch mask layer. $\mathrm{SiO}_{2}$ and $\mathrm{V}_{2} \mathrm{O}_{5}$ thin films were deposited on the V-grooved Si substrate using PECVD and sputtering. (DC, 100W, Ar $\left.\mathrm{O}_{2} 30: 11 \mathrm{sccm}, 5 \mathrm{mTorr}, 400^{\circ} \mathrm{C} 2 \mathrm{~h} 200 \mathrm{~nm}\right) ; \mathrm{VO}_{2}$ nanowires were grown by CVD without using any catalysts. Before heating the source material, the furnace tube was evacuated to about $10^{-3}$ Torr; the furnace temperature was increased at a rate of $18^{\circ} \mathrm{C} / \mathrm{min}$ to a final temperature of $\sim 750^{\circ} \mathrm{C}$ which was maintained for $2 \mathrm{~h}$. A constant flow of high purity helium $(1000 \mathrm{sccm})$ was maintained in the chamber throughout the experiment. The optimal deposition temperature was found to be between $700{ }^{\circ} \mathrm{C}$ and $750^{\circ} \mathrm{C}$. The size distribution, lattice structure, and crystal orientation of the as-synthesized products were 
characterized by scanning electron microscopy (SEM), x-ray diffraction (XRD), and transmission electron microscopy (TEM).

Fabrication of strain sensor: In order to completely separate the $\mathrm{VO}_{2}$ nanowire and the $\mathrm{SiO}_{2}$ from the $\mathrm{SiO}_{2} / \mathrm{Si}$ substrate, it was etched by BOE for 1 hour and rinsed with deionized water. The PDMS was prepared by mixing the liquid PDMS elastomer and a curing agent in the ratio 10:1 by weight. The liquid mixture was poured onto the $\mathrm{VO}_{2}$ nanowire arrays grown on the Si substrates and thermally cured at $90^{\circ} \mathrm{C}$ for 30 min. After curing, the PDMS was peeled off the $\mathrm{VO}_{2}$ nanowires array covered Si substrate, and $\mathrm{Cr} / \mathrm{Au}(20 / 300 \mathrm{~nm})$ electrodes were fabricated on the substrate across the $\mathrm{VO}_{2}$ nanowires. A constant 1 $\mathrm{V}$ was applied to evaluate the performance of the strain sensor.

Molecular dynamical simulation: Molecular dynamical simulations were performed using the open source package, GROMACS v 4.5.4. Lennard-Jones (LJ) particles were assumed to interact via a LJ potential with a cutoff at $2.5 \sigma$ and $\epsilon_{p p}=2.0 k_{B} T$. The surfaces were constructed by arranging LJ particles whose positions were fixed. The mobile LJ particles also interact with the surface particles via the LJ potential with a cutoff at $2.5 \sigma$ but with $\epsilon_{p s}=0.8 k_{B} T$. ( $k_{B}$ is the Boltzmann constant, $T$ is the absolute temperature, $\sigma$ is an approximate diameter of $\mathrm{LJ}$ particles, which were set to 1 , and $k_{B} T$ is set to 1.0 using the V-rescale method.)

Characterization: The morphologies of the $\mathrm{VO}_{2}$ nanowire was investigated using a cold field emission scanning electron microscope (FE-SEM, S-4800, Hitachi) with an accelerating voltage of $10 \mathrm{kV}$. Elemental analysis was carried out in the SEM using energy-dispersive X-ray spectroscopy (EDX). The high-resolution TEM images were collected using a Cs-corrected JEM-2100F operated at $200 \mathrm{kV}$. For EELS experiments, a dedicated scanning transmission electron microscope JEM-2100F was used. The microscope was operated at $200 \mathrm{keV}$. It is equipped with an electron energy-loss spectrometer (Gatan, Enfina). The energy resolution in EELS, as measured by the full width at half maximum of the zero-loss peak, was $0.8 \mathrm{eV}$. 

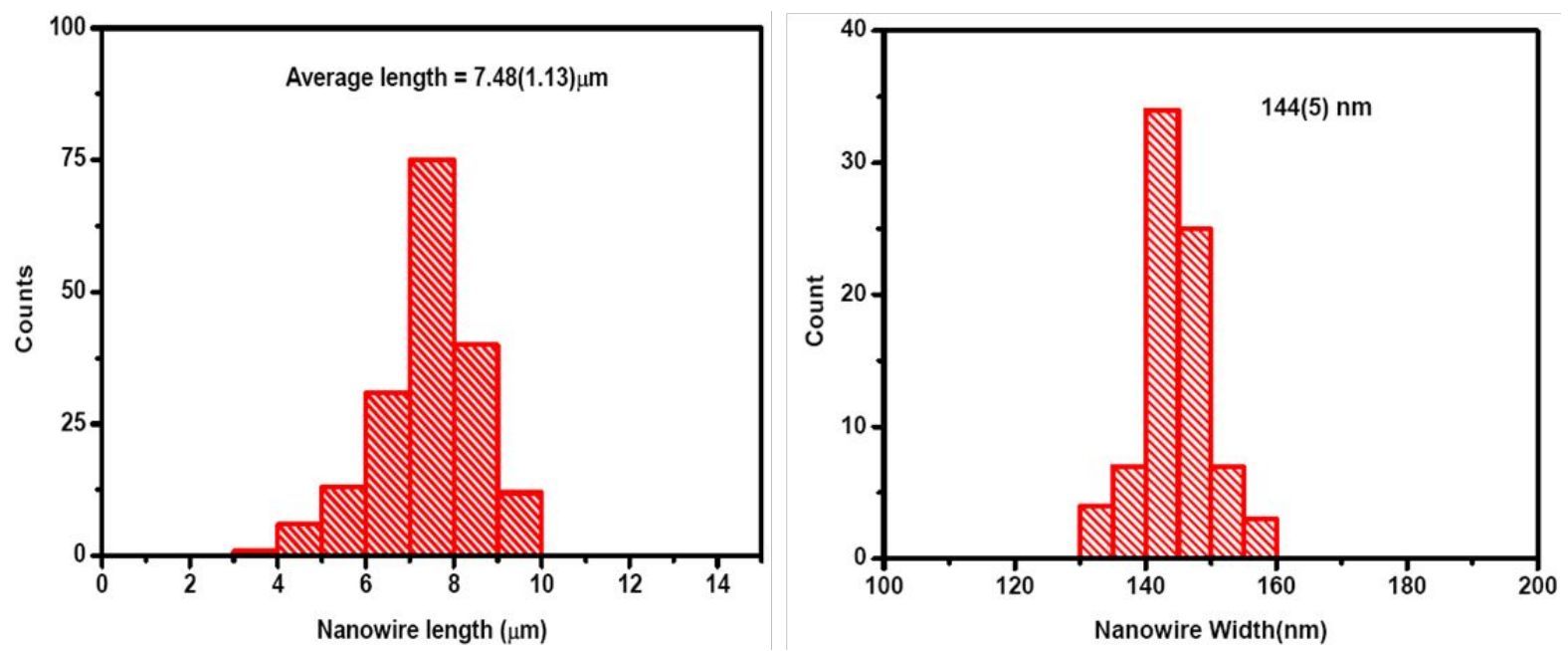

Figure S1. The histogram of length and width of randomly oriented $\mathrm{VO}_{2}$ nanowires on a $\mathrm{SiO}_{2} / \mathrm{Si}$ substrate.
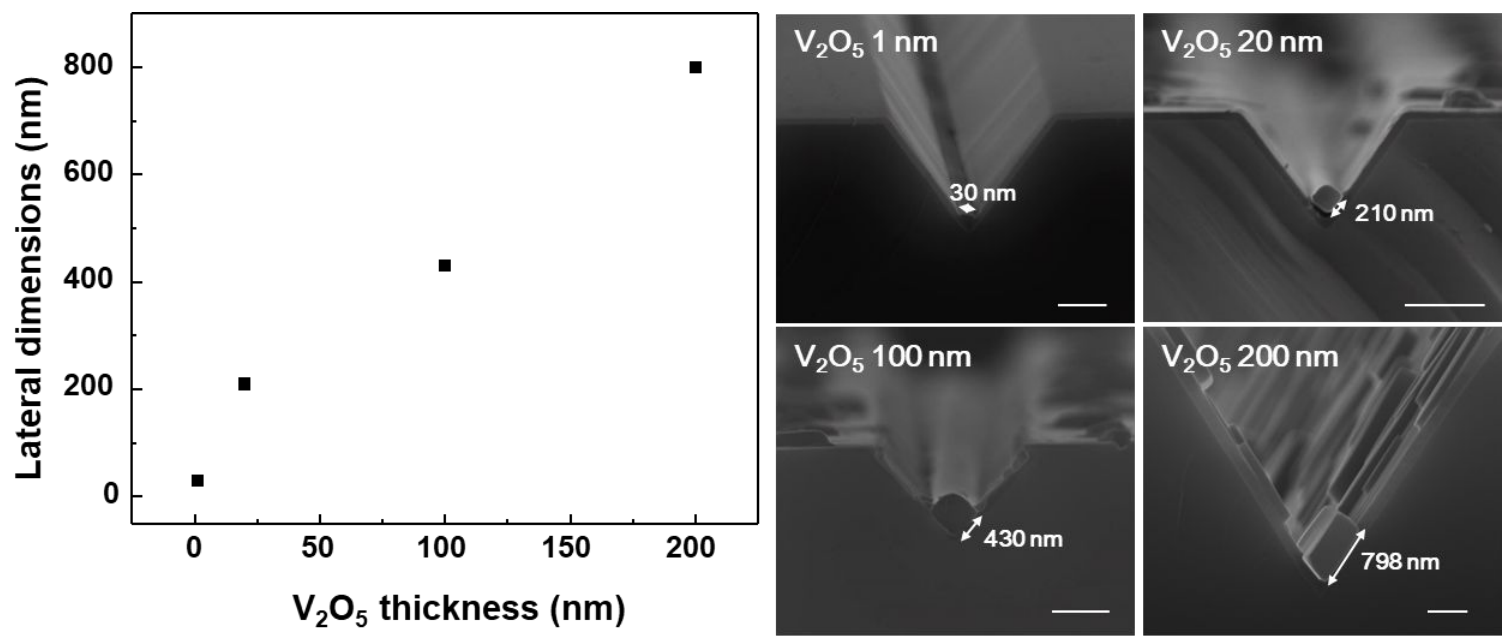

Figure S2. The $\mathrm{VO}_{2}$ nanowire growth as a function of the $\mathrm{V}_{2} \mathrm{O}_{5}$ thin film thickness. Scale bars, 
$500 \mathrm{~nm}$. 

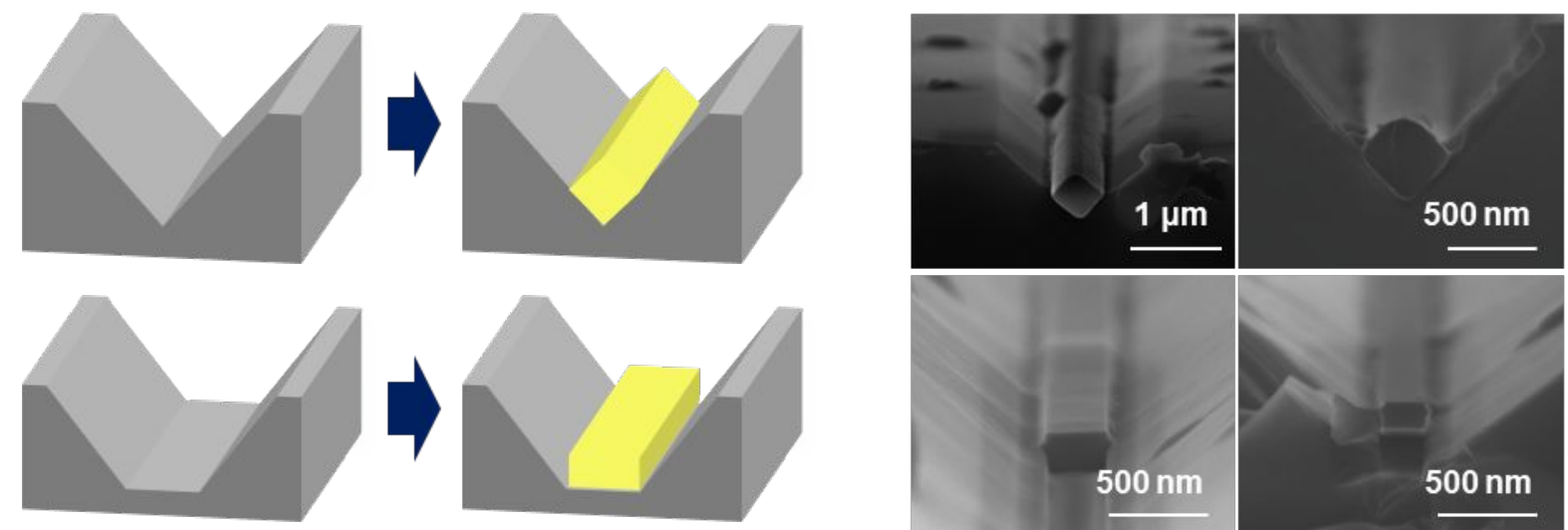

Figure S3. The cross-sectional images of the grown $\mathrm{VO}_{2}$ with the shape of the $\mathrm{V}$-grooved

\section{surface.}
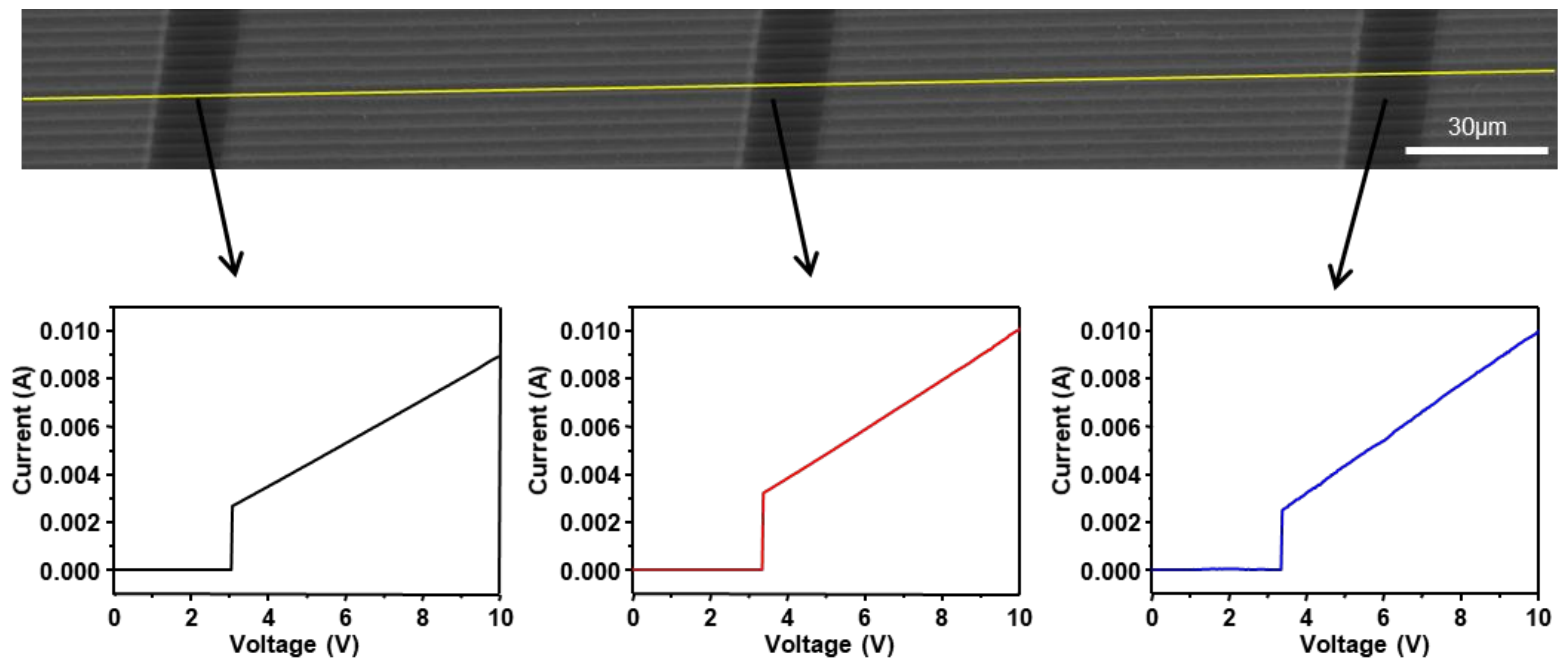

Figure S4. The millimeter-long $\mathrm{VO}_{2}$ nanowires SEM image and I-V measurement graph.

a

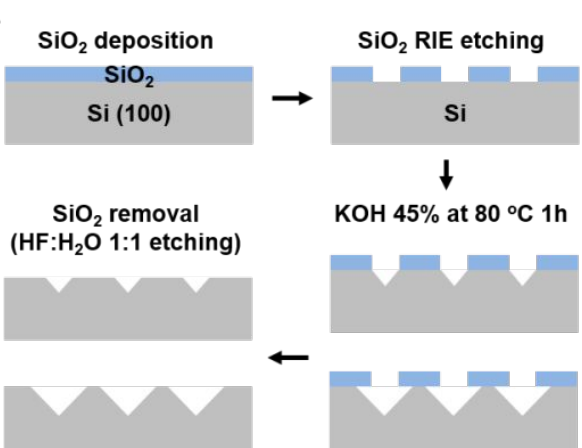

b
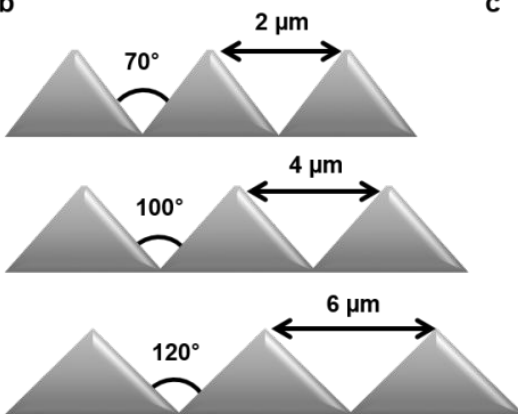

c

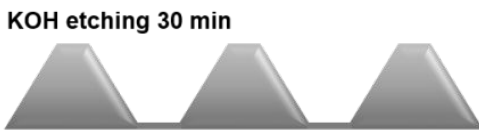

$\mathrm{KOH}$ etching $50 \mathrm{~min}$

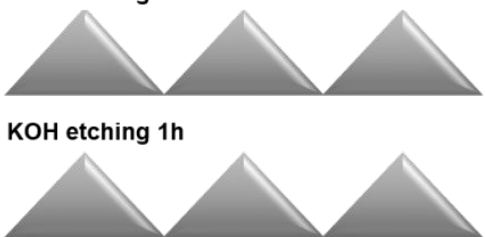

Figure S5. (a) Schematic fabrication process of V-groove templates and schematic diagrams of the $\mathrm{V}$-groove templates with different pitch size (b) and $\mathrm{KOH}$ etching time (c). 
a

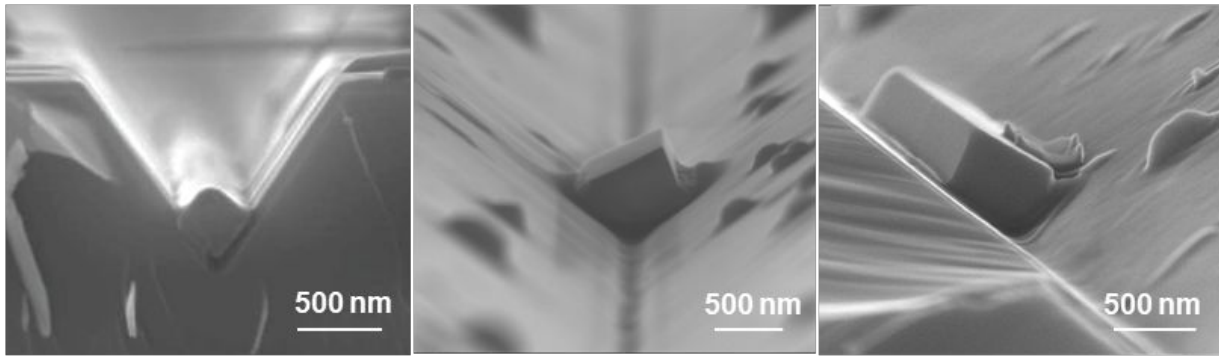

b
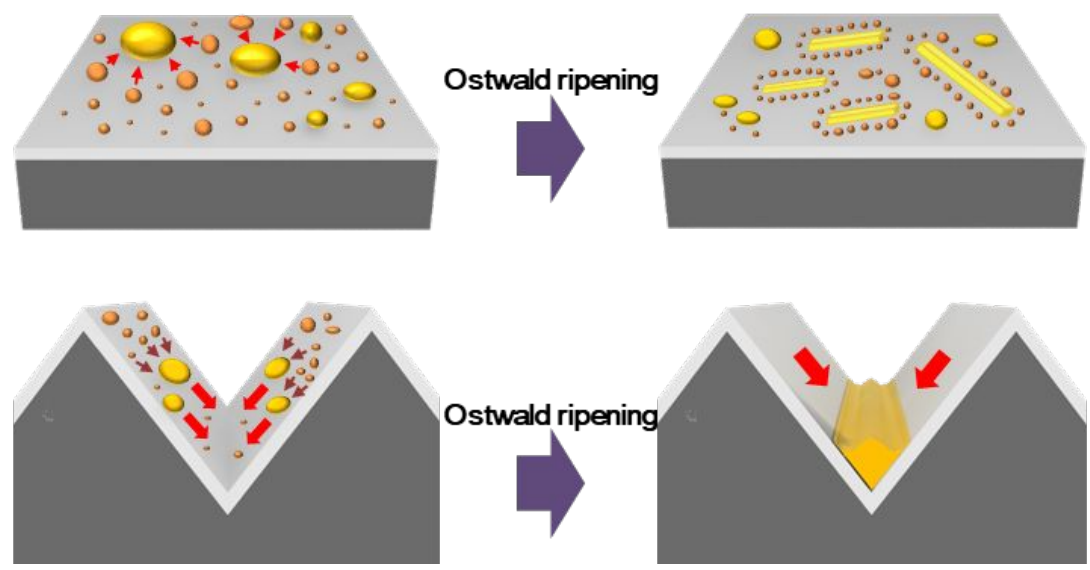

Figure S6. (a) The SEM image of $\mathrm{VO}_{2}$ nanwire tail located on $\mathrm{V}$ groove substrate and (b) the schematic image of Ostwald ripening process on flat substrate and V groove substrate.
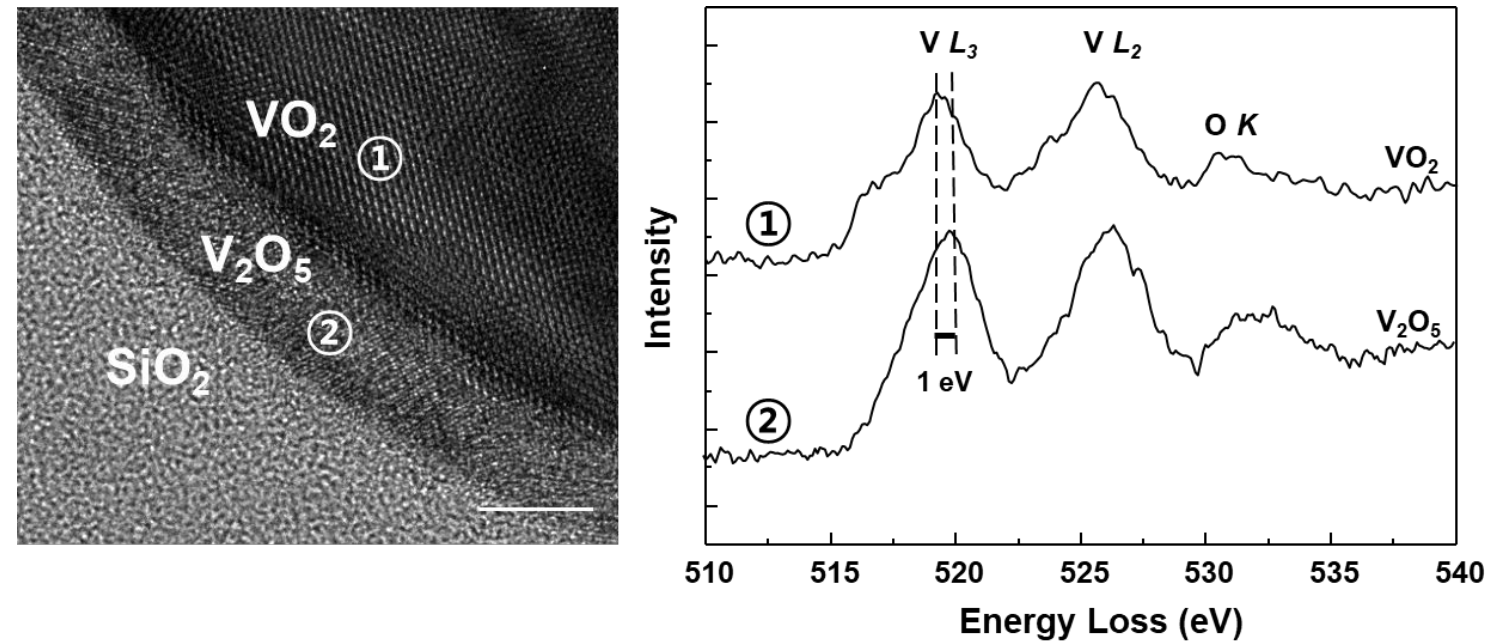

Figure S7. Electron energy loss spectroscopy (EELS) in $\mathrm{V}_{2} \mathrm{O}_{5}$ and $\mathrm{VO}_{2}$ regions. 

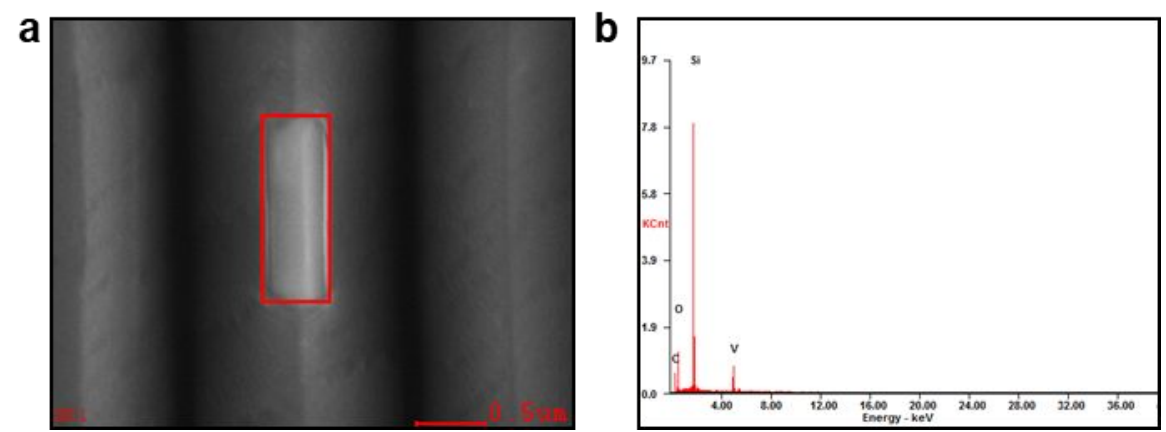

Figure S8. (a) SEM image and (b) EDXS result of the transferred $\mathrm{VO}_{2}$ nanowire onto PDMS substrate.

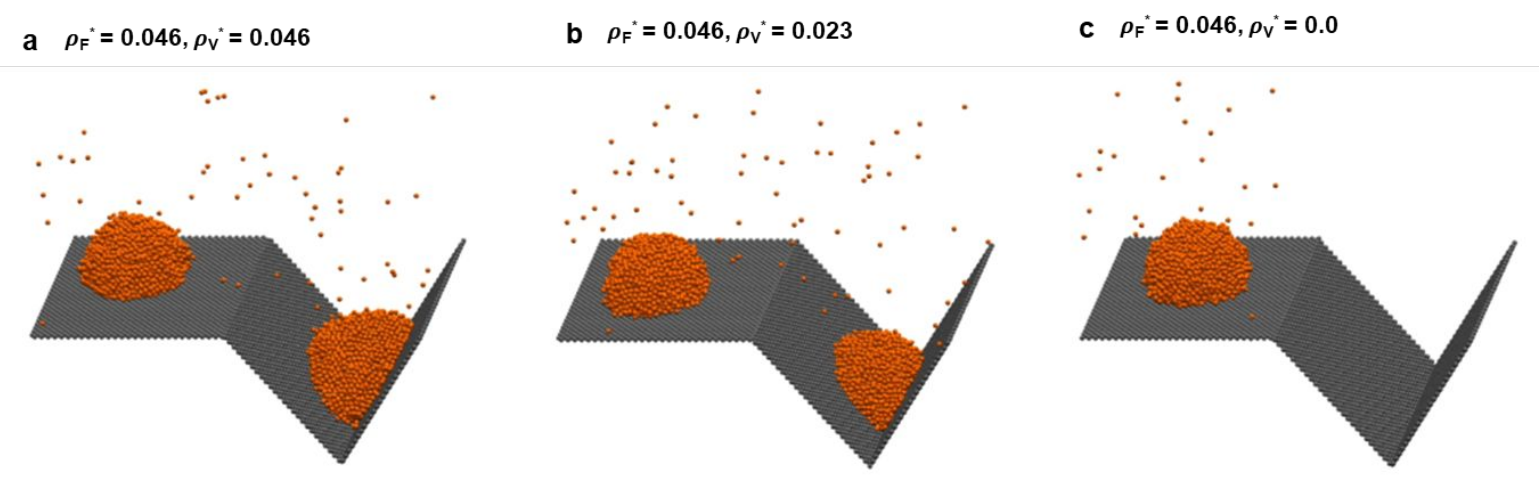

Movie 1. Video showing the results of a real-time molecular dynamics (MD) simulation of directional Ostwald ripening. 